\title{
Particle Physics Phenomenology
}

\author{
Maria Beatriz Gay Ducati \\ Instituto de Física, Universidade Federal do Rio Grande do Sul \\ Caixa Postal 15051, CEP 91501-970, Porto Alegre, RS, Brazil
}

Received on 9 January, 2004

\begin{abstract}
We give a summary of contributed talks and papers in the plenary, parallel and poster sessions of the XXIV
\end{abstract} ENFPC (Brazilian National Meeting on Particles and Fields) in the area of particle phenomenology.

\section{Introduction}

In the XXIV ENFPC were presented one plenary talk, three parallel talks, 15 oral communications and 27 posters on Phenomenology.

The research on phenomenology presented at XXIV ENFPC could be divided in some main domains: extensions of the standard model and searches; heavy quarks production, and models; high density QCD and nuclear dependence and models; perturbative and non-perturbative QCD; neutrinos, and cosmic rays.

We had presentations from researchers representing 16 brazilian institutions, from the south-east of Brazil, showing a strong regional concentration of the research in this field. Also, there were represented 12 foreign institutions, from plenary speakers to collaborators, demonstrating a high level of international interaction in our community.

The quality of oral presentations as well as the posters, and the degree of participation and discussions is increasing each year, which is one of the aspects to be considered by the funding agencies. The meeting is very relevant for MSc and $\mathrm{PhD}$ students, Pos-docs as well as for professors, and is quite representative of the area, although a higher level of participation of seniors in the field would be highly appreciated.

\section{Plenary and Parallel Talks}

In a very pedagogical plenary talk, we heard Prof. Xin-Nian Wang, from Lawrence Berkeley National Laboratory, explain the aspects of jet tomography of quark-gluon plasma [1]. The technique of jet tomography is particularly suitable for the analysis of heavy ions collisions, specially the study of the properties of the dense matter formed at Relativistic Heavy Ion Collider (RHIC). The high $p_{T}$ partons degradate during their propagation in the dense medium since multiple scattering promotes radiative energy loss. This induces a suppression on high $p_{T}$ spectra from jet fragmentation, which is known as jet quenching, already observed in $A u-A u$ collisions at RHIC. Other aspects involved in the technique are the disappearance of back-to-back jet-like hadron correlations and the finite azimuthal anisotropy of high- $p_{T}$ hadron spectra, also connected with jet quenching. The implementation of jet tomography as a tool is related, on the theoretical side, with the calculation of radiative parton energy loss, which at RHIC is 30 times higher than in cold nuclei, and with the modified fragmentation function. It would provide valuable information on dense matter physical properties and possibly to extract the initial parton density, and formation time, using the effective total parton energy loss. The initial parton density is key information to provide initial conditions which is extremely relevant to determine the most suitable evolution equation for the partonic system, meaning the dynamics of QCD.

In the parallel talk on dynamics of dense partonic systems, Victor Gonçalves, from UFPel, evolved through the theoretical and phenomenological issues concerning high density QCD [2], like saturation, and their relation with the interface between perturbative and non-perturbative QCD. It was discussed how it relates with non-linear dynamics expressed in the parton evolution equations GLR, AGL, and BK [3]: this is not closed subject, and several related issues to provide information on initial conditions where addressed in the above plenary talk. It was also presented an interesting contribution of geometric scaling at DIS through the analysis of charm production at HERA.

We heard on KamLand data and the solution to the solar neutrino problem, by Pedro de Holanda, from UNICAMP [4]. The KamLand results present good agreement with predictions based on solar neutrino data. The result suppress positive sign oscillation and eliminates the solution of no oscillation. The best fit combining 84 experimental data from 5 experiments gives $\Delta m^{2}=7.5 \times 10^{-5}$ $\mathrm{eV}^{-2}, \sin ^{2} 2 \theta=0.9$. The best fit splits LMA (Large Mixing Angle) region, and the low mass LMA is preferred, $\Delta m^{2}<10^{-4} \mathrm{eV}^{-2}, 99 \% \mathrm{CL}$, and predict for $S N O$ : $C C / N C=0.33+0.05-0.03$.

The parallel talk presented by Pedro Galli Mercadante, from USP [5] , addressed the search for supersymmetry at the Large Hadron Collider (LHC). The investigation concerns SUSY at electroweak scale which analysis at ATLAS and CMS is on the region of large scalar masses. It is a search for new physics at $\mathrm{TeV}$ scale and in the simplest 
scenario, MSSM involves two superpartners $\left(m_{H}<140\right.$ $\mathrm{GeV}$ ). Since for the Tevatron the limit for gaugino mass is $m_{1 / 2}<200 \mathrm{GeV}$, at LHC this limit goes to $m_{1 / 2} \leq 900$ $\mathrm{GeV}$, and for the supersymmetric quarks $m_{\text {squarks }}<2$ $\mathrm{TeV}$, and $m_{\text {gluino }}(G M S B)<2.9 \mathrm{TeV}(0.9 \mathrm{TeV}$ at Tevatron). LHC has the potential to cover natural region in many SUSY scenarios and it is very exciting motivation for those searches.

\section{Oral Communications and Posters}

To summarize all the talks from parallel sessions and posters I adopted a personal point of view to separate and classify the works following what I considered their main subject, mostly related with the main domains mentioned in the Introduction. Obviously, this positioning has only the goal to systematize this review: a paper in several cases pertains to more than one subject.

We can identify a significant amount of work related with extensions of the Standard Model and the searches of new physics in new fundamental energy scales, still divided in some main subjects.

Several authors dedicated effort on the 3-3-1 model [6], which is a robust model for the physics at $\mathrm{TeV}$ scales with 3 triplets to generate lepton masses. Besides coinciding with the standard model at low energy, the model provides $\mathcal{N}=1$ SUSY at energy scale $\mu(\approx 4 \mathrm{TeV})$. In the contribution by A. G. Dias, C. A. de Sousa Pires, P. S. R. da Silva [7], it is shown that the global symmetries are satisfied in 3-3-1 with right-handed neutrinos. The breaking of Peccei-Quinn symmetry yields an invisible axion, as a candidate of cold dark matter (CDM). An another characteristic of 3-3-1 model is the existence of double charged Higgs boson, which was studied by J.de Sá Borges, M.D. Tonasse and J.E.C. Montalvo, through production and signatures at LHC in Drell-Yan and gluon-gluon processes [8]. Aiming to keep the axion protected from gravitational effects, and automatically include Peccei-Quinn in 3-3-1 models, A.G. Dias and V. Pleitez [9] implemented local $Z_{N}$ symmetries with $N=11$ and 13 . The supersymmetric 3-3-1 model is employed by J.C. Montero, V. Pleitez, M.C. Rodrigues to calculate the neutralinos and charginos masses, and to emphasize the difference in the generation of lepton mass production mechanism with the usual 3-3-1 and the minimal SUSY model [10].

The dynamical symmetry breaking mechanism through a small coupling constant and a dynamical scale, is employed to generate masses for the first and third generation of fermions, respectively related to the dynamical quark mass $(\approx 250 \mathrm{MeV})$ and the dynamical techniquark mass $(\approx$ $250 \mathrm{TeV})$. This issue of technicolor model was presented by A. Doff and A. Natale [11].

The search for new charged leptons and signatures in next generation of ep $(\sqrt{s}=500 \mathrm{GeV})$ colliders was addressed by F. M. L. de Almeida Junior, Y. A. Coutinho, J. A. M. Simões, S. Wulck, M.A.B. do Vale . The final state leptonic distribution was shown to be particularly useful for the purpose of a signature of the heavy charged leptons [12].

Higgs search at LHC is main issue, and studied in weak boson fusion channel with decay into weak boson pairs pro- ved to be robust for extensions of the SM and for MSSM with several higgs doublets, by A. Alves, O. Eboli, T. Plehn and D. Rainwater, who also emphasized transverse mass distribution to distinguish higgs from SM [13].

LHC potential to reach the focus point region in $\mathrm{mSU}$ GRA parameter space, consistently with WMAP results, meaning multi-TeV masses for squarks and sleptons, is explored by b-tagging for large $E_{T}$ gluino decays by P. G. Mercadante, J. K. Mizukoshi [14].

The subject of heavy quarks production, distribution and models, which is a source of extensive phenomenology, and counts with very good data in several cases, was addressed in several contributions.

The Color Evaporation Model describes well the data from DELPHI for $\gamma \gamma \rightarrow J / \Psi+X$, moreover the transverse momentum distribution of $J / \Psi$, as shown by O. Éboli, J.K. Mizukoshi and E.M. Gregores [15]. Quarkonia is also analysed by C.B. Mariotto, MBGD and G. Ingelman, through the $x_{F}$ and $p_{T}$ distributions of bottomonium in hadronic collisions, using soft color models and pQCD, covering fixedtarget limits and also giving high energy predictions [16]. The recombination mechanism, as opposite to the fragmentation, is considered to explain the large $x_{F}$ behavior of the charm production in $\pi N$ in the work by J.E. Magnin, C. Ávila, L.M. Mendoza-Navas [17]. The $J / \Psi$ cross section in elastic photo and electron-production is calculated nonperturbatively, employing the stochastic vacuum model, and also $\sigma_{L} / \sigma_{T}$, and $Q^{2}$ distributions, with good agreement, by E. Ferreira, H.G. Dosch [18]. Inclusive charm production at HERA ep collider provides the study of large range of photon virtuality $\left(Q^{2}\right)$, however the saturation momentum $Q_{\text {sat }}^{2}(x)$ ranges below the hard scale $\left(\mu_{c}^{2}=4 m_{c}^{2}\right)$. This aspect is explored, using two saturation models, showing a scaling $\tau=\frac{Q_{0}^{2}}{Q_{\text {sat }}^{2}(x)}$, above the saturation limit, meaning a geometric scaling regime, in the work by V. P. Gonçalves, M.V.T. Machado and M.M Machado [19] The control channel $B_{d}^{0} \rightarrow J / \Psi k^{+0}$ is proposed and analysed by L.S. de Paula and E.C. de Oliveira, in order to study systematics for the performance of the $\mathrm{LHCb}$ detector, including final state acceptance [20].

Heavy ion collisions, and $A$-dependence in high density QCD are very relevant subjects in the last years, partly motivated by the available and future data, mostly from RHIC, and the general interest on the possibility of the formation of the quark gluon plasma, as well as the aspect of saturation of the gluon distribution function required by unitarity. Several works addressed the rich phenomenology with nucleus from the high energy point of view.

Among them, some authors dedicated their interests to peripheral and ultraperipheral collisions. The calculation using saturation in the color dipole picture in two photon process from heavy ion ultraperipheral collisions, enhances the heavy quark production cross section following the work by V.P. Gonçalves and M.V.T. Machado [21]. The same authors considered two-photon process also to obtain double meson production in $A-A$, for RHIC and LHC, obtaining that the mechanism is a good discriminator among different proposed QCD dynamics [21]. The phenomenological saturation model is applied to deeply virtual Compton scattering, 
at HERA, and is also analysed the inclusion of the real amplitude sector and skewedness, meaning non-diagonal partonic distributions in the contribution by M. V. T. Machado and L. Favart [22] Also the photon fusion process is employed by C.G. Roldão and A.A. Natale to calculate the $\mu^{+} \mu^{-}$, and $\tau^{+} \tau^{-}$cross sections in peripheral collisions, for RHIC and LHC, analysing the variation with energy dependence on the nuclear radius [23].

$J / \Psi$ production at RHIC and LHC is studied, including energy loss and saturation of partonic distribution function considering parton recombination, and multiple scatterings of the $c \bar{c}$ with the medium, comparing with NA50, data, and giving predictions for higher energies, in the work by MBGD, L.F. Mackedanz and V.P. Gonçalves [24]. Also including saturation mechanism, although through an extension of the Glauber-Gribov formalism, larger nuclear heavy quark cross sections for LHC energies are obtained by V.P. Gonçalves and M.V.T. Machado [25]. The behavior of partonic distributions in dense medium on the virtuality $\left(Q^{2}\right)$ is compared in the Glauber-Mueller and DGLAP formalisms for the dynamics in $e A$ processes, by A. L. Ayala Filho, V. P. Gonçalves, D. R. Gratieri [26].

A diluted system of gluons named Color Glass Condensate (CGC) [27] has been proposed to describe large gluon density in an extensive nuclear medium. The saturation phenomena through this formulation is associated with a scale $Q_{s}^{2}(x, A)$. Although not still determined precisely, the scale can be approximated by the study of the dilepton transverse momentum $p_{T}$ distribution, since at low $p_{T}$ large effects are expected. This is shown for RHIC and LHC in the work by M.A. Betemps and MBGD [28]. A phenomenological parametrization for the color field correlator in the medium proposed in CGC is presented, allowing to calculate photoproduction of charm and bottom, at LHC energies, in ultraperipheral collisions, in the work by V. P. Gonçalves and M.V.T. Machado [29]

Spin-1 particles are investigated in the light front field theory, in the work by J. P. B. C de Melo and it is shown that the covariance of the eletromagnetic current is restored by the inclusion of the zero modes, then overcoming the rotational symmetry breaking problem [30]. The same LF model is applied to the semileptonic decays of hyperons and heavy barions by the construction of an effective lagrangian considering scalar, vector and mixed spin couplings schemes among the quarks, in the contribution by E. F. Suisso, T. Frederico, M. Beyer and H. J. Weber [31].

The connection between observables in ultra relativistic heavy ion collision and thermodynamical variables was the object of the work of C. E. Aguiar, T. Kodama, L. L. S. Portugal and A. G. Grunfeld. The authors express founded worry on the usually employed idea of global statistical equilibrium based on grand canonical ensembles, proposing instead the use of Isobaric (Iso) ensemble, more appropriated for finite systems [32]. It is shown that hydrodynamical instabilities are not limited by the chiral critical point, by the study of chiral and pressure waves on the onset of unstable modes, this enlarged domain having implications in heavy ions collisions and models for the early universe, following C. E. Aguiar, E. S. Fraga and T. Kodama [33]
Aiming to access nonperturbative region in QCD, through the solution of the Schwinger-Dyson equations, conducts to some solutions with frozen value of the coupling constant. This value is related with the $\beta$ function (RG for pure non-Abelian theory) fixed point once dynamical gluon mass scale is invoked to satisfy the global minimum of energy, as presented by A. C. Aguilar, A. A. Natale, P. S. R. da Silva [34]. A novel regularization scheme is applied by R. L. S. Farias, O. A. Battistel, G. Krein to the finite NambuJona-Lasinio model, in order to deal with divergent amplitudes, which is free of ambiguities and symmetry violations . The method is shown to be appealing since it allows a single mass scale to treat the divergent integrals in chiral symmetry breaking in connection with charge independence [35]. Phase-shift definitions in chiral perturbation theory and in Unitarization Program applied to pion-nucleon amplitudes moved the analysis of J. S. Borges Filho, I. P. Cavalcante, showing that UP amplitudes are more efficient to fix parameters, since less sensitive than ChPT [36].

The pions and kaons parton distribution function are studied by J. C. Sanabria, C. Avila and J. Magnin who present a parametrization for $\pi\left(x, Q^{2}\right)$ and $K\left(x, Q^{2}\right)$, fizing parameters using E615 and NA63 data [37].

Pentaquarks in the QCD sum rule framework were proposed to explain the state $\Theta^{+}(1540)$ as $[\bar{u} d]^{2} \bar{s}$, with good agreement, also suggesting the state $[\bar{u} d]^{2} \bar{d}$ to be compatible with $N(1440)$, the Roper resonance, following the work by R.D. Matheus, F.S. Navarra, M. Nielsen, R. Rodrigues da Silva and S.H. Lee [38].

The interest in neutrino physics in our community is steadly increasing, with solid established collaborations and several relevant contributions.

Besides the already mentioned parallel talk [4] the contributions ranged from an introductory motivational review of flavor oscillation in vacuum and matter by G. A. Valdiviesso and M. Guzzo [39] to a contribution on detection of very high energy neutrinos. In this work by C. A. de Moura Junior and M. Guzzo [40], it is shown the observational potential of the Pierre Auger Observatory to detect $\nu_{\tau}$ with $E_{\nu_{\tau}} \approx 1 \mathrm{EeV}$, from Double Bang events, meaning the interaction in the atmosphere of very high energy $\nu$ from extragalactic sources, providing in some cases a second shower from $\tau$ decay.

Considering a simulation of the conditions of experiment LSND, the oscillation $\hat{\nu}_{\mu}-\hat{\nu}_{e}$ is calculating in a two families, and three families of neutrinos framework by $\mathrm{C}$. Frigério, V. M. de Aquino [41] with the goal to conciliate all available data.

The requirement of a heavy neutrino in see-saw mechanism is examined by R. S. Gomes and F. M. L. de Almeida Júnior concerning the possibility of detection in ATLAS of heavy Majorana neutrinos, basically $q_{i} q_{j} \rightarrow N_{e} \rightarrow e^{ \pm} e^{ \pm} j j$ [42].

Solar neutrino problem solution known as LMA, related to the neutrinos oscillation enhanced by matter, is sensitive to randomic perturbations of the solar matter allowing $\mathrm{M}$. Guzzo, P. C de Holanda and N. Reggiani to show new regions of interest for KamLAND, the very low LMA [43].

The consideration of the particle rigidities in the cosmic 
ray spectrum implies heavier composition above the knee of the spectrum and produces suppression of very high energy, $E_{\nu}>10^{14} \mathrm{eV}$, diffuse neutrino fluxes, and consequently their detection would be more difficult by the work by $\mathrm{J}$. Candia, E. Roulet [44].

Combining the Earth velocity with respect to the cosmic background radiation with the neutrino velocity distribution generates a neutrino wavelength variation, which is mass dependent, once detected on Earth, . This provides a new method of determination of the different neutrino masses by the detection of the cosmic $\nu$ background, as proposed by M. Makler and J. Rafelski [45].

The reaction channel $\gamma \gamma \rightarrow \pi^{0} \rightarrow \nu_{R} \bar{\nu}_{L}\left(\nu_{L} \bar{\nu}_{R}\right)$ has its rate strongly suppressed in dense hadronic matter due to multi-particle effects, for comprehensive analysis of righthanded $\nu_{s}$, massive left-handed $\nu_{s}$ and massless $\nu_{L}^{\prime} s$. However, this channel is the most efficient $\nu$ cooling process, applicable to neutron stars, in the color-flavor-locked superconducting phase, following the contribution by F. Arretche, A. Natale and D. N. Voskresensky [46].

A calculation of muon fluxes and muon charge ratio was presented for energies from a few $\mathrm{GeV}$ to several $\mathrm{TeV}$ and Zenith angles from $0^{\circ}$ to $89^{\circ}$, applying depth-like ordered exponential operator to solve the hadron diffusion equations. The authors H. Portella, L. C. S. Oliveira, C. E. C. Lima, got very good agreement with data at sea level [47].

The fascinating project of the Extreme Universe Space Observatory (EUSO) designed to operate in space, to observe and measure extensive air showers initiated by Ultra High Energy Cosmic Rays (UHECR's) was presented by J. A. Muniz, R. Engel, J. A. Ortiz, T. K. Gaisser and T. Stanev [48]. Besides the high statistics expected, it contemplates the possibility to detect $\nu$ events above $10 \mathrm{EeV}$.

\section{Concluding Remarks}

The XXIV ENFPC was an exciting meeting showing more maturity of the phenomenology area, although the regional concentration and stability of the number of contributions. The works presented comprehend 120 authors from Brazilian institutions, 15 published papers, 8 submitted and/or in the web, 19 in progress, as informed in the contributions.

We may ask where are we going, and as far as main subjects presented it is noticeable the research on extensions of the SM and searches, neutrino physics as a whole and very high energy cosmic rays, nonlinear phenomena on high density QCD, and perturbative and nonperturbative calculations on QCD analised from different aspects. I understand there is room to improve the effort on astroparticle physics in general as well as statistical methods in dense matter.

\section{Ackowledgement}

I would like to thank the Organizing Committee for the kind invitation to present the summary talk on phenomenology. It was an excellent opportunity to have a deeper knowledge of the scientific interests of our community. I apologize in advance if involuntarily one of the contributions was not mentioned in this review.

\section{References}

[1] X.-N. Wang, Nucl. Phys. A 715, 787 (2003); Nucl. Phys. A 702, 238 (2002); these proceedings.

[2] V. P. Gonçalves, Braz. Jour. Phys. 34, 1406 (2004).

[3] L. V. Gribov, E. M. Levin, and M. G. Ryskin, Phys. Rep. 100, 1 (1983). A. L. Ayala, M. B. Gay Ducati, and E. M. Levin, Nucl. Phys. B 493, 305 (1997); Nucl. Phys. B 511, 355 (1998). Y.V. Kovchegov, Phys. Rev. D 60, 034008 (1999); Phys. Rev. D 61, 07418 (2000) I. Balitsky, Nucl. Phys. B 463, 99 (1996).

[4] P. de Holanda and A. Yu. Smirnov, Braz. Jour. Phys. 34, 1416 (2004).

[5] P. G. Mercadante Braz. Jour. Phys. 34, 1424 (2004).

[6] A. G. Dias, V. Pleitez, and M. D. Tonasse Phys. Rev. D 67, 095008 (2003).

[7] A. G. Dias, C. A. de S. Pires, and P. S. R. da Silva, Phys. Rev. D 68, 115009 (2003).

[8] J. de Sá Borges, M.D. Tonasse, and J.E.C. Montalvo, poster P081 in the XXIV ENFPC.

[9] A. G. Dias and V. Pleitez, arXiv:hep-ph/0308037.

[10] J. C. Montero, V. Pleitez, and M. C. Rodriguez, oral communication $\mathrm{CO} 04$ in the XXIV ENFPC.

[11] A. Doff, A. Natale, Phys. Rev. D 68, 077702 (2003).

[12] F. M. L. de Almeida Junior, Y. A. Coutinho, J. A. M. Simões, S. Wulck, and M.A.B. do Vale, Eur. Phys. J. C, 30, 327 (2003).

[13] A. Alves, O. Éboli, T. Plehn, and D. Rainwater, ePrint arXiv:hep-ph/0309042.

[14] P. G. Mercadante and J. K. Mizukoshi, oral communication CO13 in the XXIV ENFPC.

[15] O. Éboli, J.K. Mizukoshi, and E.M. Gregores, Phys. Rev. D 68, 094009 (2003).

[16] C. B. Mariotto, M. B. Gay Ducati, and G. Ingelman, oral communication CO013 in the XXIV ENFPC.

[17] J.E. Magnin, C. Ávila, L.M. Mendoza-Navas, ePrint arXiv: hep-ph/0307358.

[18] E. Ferreira, H.G. Dosch, Phys. Lett. B 576, 83 (2003).

[19] V. P. Gonçalves and M. V. T. Machado, Phys. Rev. Lett. 91, 202002 (2003).

[20] L.S. de Paula, E.C. Oliveira, poster P085 in the XXIV ENFPC.

[21] V.P. Gonçalves, M.V.T. Machado, Eur. Phys. J. C 28, 71 (2003); Eur. Phys. J. C 29, 271 (2003).

[22] M. V. T. Machado, L. Favart, Eur. Phys. J. C 29, 365 (2003).

[23] C.G. Roldão, A.A. Natale, ePrint arXiv:nucl-th/0310050.

[24] M. B. Gay Ducati, L.F. Mackedanz, and V.P. Gonçalves, ePrint arXiv:hep-ph/0306129.

[25] V. P. Gonçalves, M. V. T. Machado, Eur. Phys. J. C 29, 37 (2003).

[26] A. L. Ayala Filho, V. P. Gonçalves, and D. R. Gratieri, poster P071 in the XXIV EFPC. 
[27] L. McLerran, R. Venugopalan. Phys. Rev. D 49, 2233 (1994); ibid. 49, 3352 (1994). J. Jalilian-Marian., A. Kovner, A. Leonidov, and H. Weigert. Nucl. Phys. B 504, 415 (1997); Phys. Rev. D 59, 014014 (1999).

[28] M. A. Betemps, M. B. Gay Ducati, poster P080 in the XXIV ENFPC.

[29] V. P. Gonçalves, M. V. T. Machado, ePrint arXiv:hep$\mathrm{ph} / 0307129$.

[30] J. P. B. C. de Melo, oral communication CO13 in the XXIV ENFPC.

[31] E. F. Suisso, T. Frederico, M. Beyer, and H. J. Weber, oral communication CO04 in the XXIV ENFPC.

[32] C. E. Aguiar, T. Kodama, L. L. S. Portugal, and A. G. Grunfeld, poster P069 in the XXIV ENFPC.

[33] C. E. Aguiar, E. S. Fraga, and T. Kodama. ePrint arXiv:nuclth/0306041.

[34] A. C. Aguilar, A. A. Natale, and P. S. R. da Silva. Phys. Rev. Lett. 90, 152001 (2003).

[35] R. L. S. Farias, O. A. Battistel, and G. Krein, oral communications CO13 in the XXIV ENFPC.

[36] J. de Sá Borges, I. P. Cavalcante, poster P073 in the XXIV ENFPC.
[37] J. C. Sanabria, C. Avila, and J. Magnin, Phys. Rev. D 67, 034022 (2003); Phys. Rev. D 68, 079902 (2003).

[38] R.D. Matheus, F.S. Navarra, M. Nielsen, R. Rodrigues da Silva, and S.H. Lee, ePrint arXiv:hep-ph/0309001.

[39] G. A. Valdiviesso, M. Guzzo, poster P072 in the XXIV ENFPC.

[40] C.A. de Moura Júnior, M. Guzzo, poster P065 in the XXIV ENFPC.

[41] C. Frigério, V. M. Aquino, poster P068 in the XXIV ENFPC.

[42] R. S. Gomes, F. M. L. de Almeida Júnior, poster P070 in the XXIV ENFPC.

[43] M. Guzzo, P. Holanda, and N. Reggiani, Phys. Lett. B 569, 45 (2003)

[44] J. Candia, E. Roulet, JCAP 0309, 005 (2003).

[45] M. Makler, J. Rafelski, poster P075 in the XXIV ENFPC.

[46] F. Arretche, A. Natale, and D. N. Voskresensky, poster P084 in the XXIV ENFPC.

[47] H. Portella, L. C. S. Oliveira, and C. E. C. Lima, poster P057 in the XXIV ENFPC.

[48] J. A. Muniz, R. Engel, J. A. Ortiz, T. K. Gaisser, and T. Stanev, oral communication CO09 in the XXIV ENFPC. 